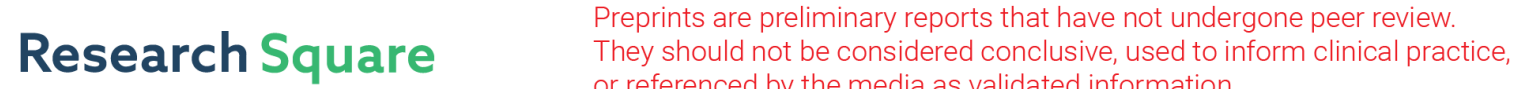 or referenced by the media as validated information. \\ Hydroxypropylation Reduces Gelatinization Temperature of Corn Starch for Textile Sizing
}

\section{Yanqin Shen}

Xian Polytechnic University: Xi'an Polytechnic University

Yijun YAO ( $\nabla$ yaoyj@xpu.edu.cn )

Xi'an Polytechnic University

\section{Zhongliang Wang}

Xi'an Polytechnic University

Hailiang Wu

Xi'an Polytechnic University

\section{Research Article}

Keywords: hydroxypropyl starch, gelatinization temperature, solubility, size film property

Posted Date: February 15th, 2021

DOl: https://doi.org/10.21203/rs.3.rs-192851/v1

License: (c) (i) This work is licensed under a Creative Commons Attribution 4.0 International License. Read Full License

Version of Record: A version of this preprint was published at Cellulose on April 7th, 2021. See the published version at https://doi.org/10.1007/s10570-021-03852-4. 


\section{Hydroxypropylation reduces gelatinization temperature of 2 corn starch for textile sizing}

3 Yanqin Shen ${ }^{\text {a,b }}$, Yijun Yao ${ }^{\text {a,b }}$, Zhongliang Wang a , Hailiang Wu ${ }^{\text {a,b }}$

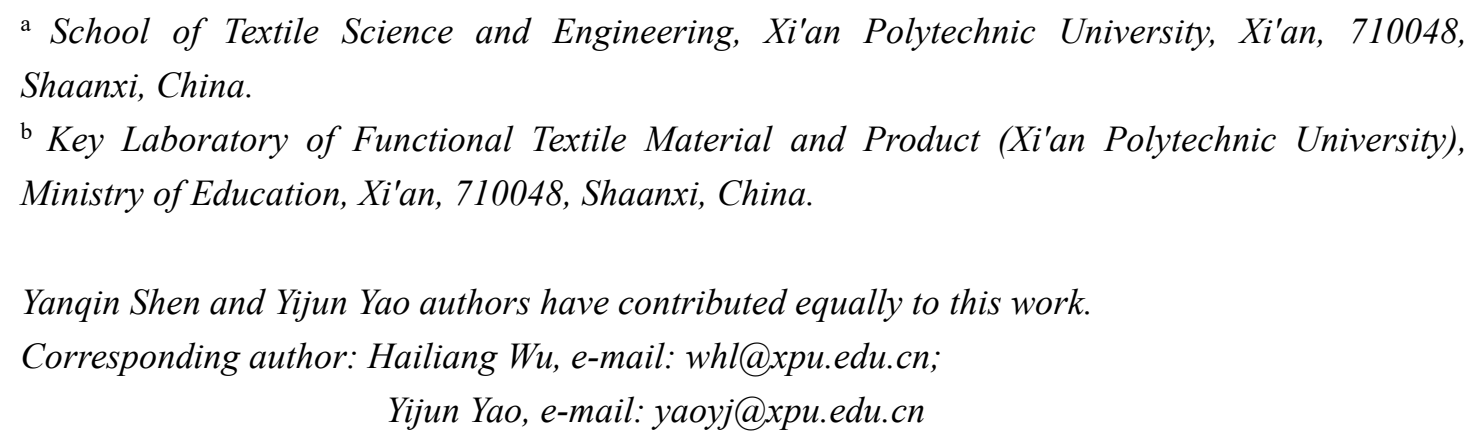

\section{Abstract}

A series of hydroxypropylated starch (HPS) that can be dissolved in water at $60-65^{\circ} \mathrm{C}$ was obtained via two-step method in water system from corn starch. The structure and property of the HPS and its gelatinization temperature were characterized by Fourier transform infrared spectrometer (FTIR), nuclear magnetic resonance spectroscopy ( ${ }^{1} \mathrm{H}$ NMR), X-ray diffraction (XRD), differential scanning calorimetry (DSC), scanning electron microscopy (SEM), and transmission electron microscope (TEM). It was concluded that hydroxypropyl mainly bonded on the hydroxyl group at $\mathrm{C}_{2}$ position from anhydroglucose unit of starch in the form of C-O-C, and the substitution level at $\mathrm{C}_{6}$ position was slightly higher than that at $\mathrm{C}_{3}$ position; and the crystallinity of starch decreased from $52.41 \%$ to $29.4 \%$ due to the introduction of hydroxypropyl and was confirmed by XRD. At the same time, the grooves on the surface of starch granules were observed by SEM. The above-mentioned two synergism promoted the permeation and transmission of water molecules in the starch microstructure. Moreover, the gelatinization temperatures and enthalpy of synthetic HPS was lower than that of raw corn starch, as further confirmed by DSC. This caused the HPS with a molar substitution greater than 0.1 soluble in water at $65^{\circ} \mathrm{C}$, and the dissolution state was similar to that of at $95^{\circ} \mathrm{C}$ (transmittance above $55 \%$ ), as well as exhibited high slurry stability. Interestingly, compared with the raw starch, the HPS film showed excellent mechanical property at the relative humidity of $65 \%$, which could be attributed 
to the hydrophilic ether bond and the flexible alkyl chain bonded on the structure of starch. This study will provide a new way for the preparation of high performance starch size for sizing yarn at medium low temperature.

Keywords: hydroxypropyl starch, gelatinization temperature, solubility, size film property

\section{Graphic abstract}

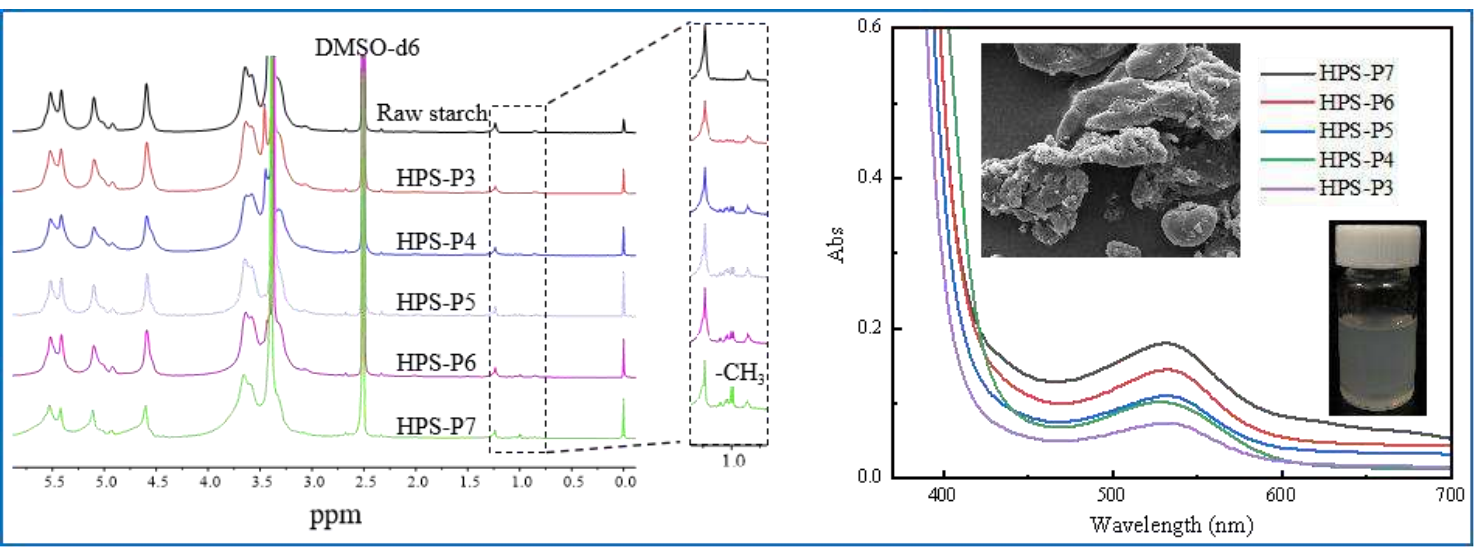

\section{Introduction}

As an abundant natural polysaccharide in plants, starch has become the most widely sizing agent in textile sizing, with accounting for about $70 \%$ of the total slurry in China due to its renewability, biodegradability and ease of modify, etc (Jeong et al. 2018; Li et al. 2016; Yang et al. 2018; Li et al. 2018a). However, the strong hydrogen bond network structure in the intra- and inter-molecular of starch and its high vitrification transition temperature, which make it can only be gelatinized to form slurry at a higher temperature, leading to a high-temperature sizing technology have been developed widely for warp sizing so far (the boiling temperature of slurry must be $100-132{ }^{\circ} \mathrm{C}$, and the slurry tank temperature has exceeded $\left.92{ }^{\circ} \mathrm{C}\right)(\mathbf{Z h u}$ et al. 2015; Wu et al. 2020). High-temperature sizing has seriously affected the working environment of workers and brought high energy consumption, which inhibit the development of the textile industry (Jiang et al. 2019; Rafikov et al. 2020). Hence, our objective is to develop a low energy-consumption and environmentally friendly sizing method at medium temperature.

As room temperature soluble textile size, polyacrylic acid polymers exhibit strong sizing effect on chemical fibers (such as polyester and nylon). However, they 
suffer the problem of moisture absorption and re-adhesion due to their inherent hydrophilic groups and flexible molecular chains, leading to they can only be used as auxiliary sizing on yarns, and their insufficient adhesion to cellulose fibers (Hu and Babu 2007). Although there are also studies on starch grafted acrylic acid (Li et al. 2011), acrylamide (Wang et al. 2020) and methacrylic acid (Djordjevic et al. 2019), the starch graft copolymers have improved sizing properties, such as grafted starch size film properties and strong adhesion to cellulose fibers. Nevertheless, the disadvantages of starch sizing are not completely eliminated, most studies focused on the synthesis of grafted copolymers and the use of various starches in the sizing of textiles, as well as the use of these modified starch size still need to be dissolved at high temperature (above $85^{\circ} \mathrm{C}$ ).

In recent years, the way of medium temperature sizing was realized mainly by developing water-soluble starch at medium temperature of $60^{\sim} 65^{\circ} \mathrm{C}$ (Xu et al. 2016; Kim et al. 2017). For example, Guo et al. have realized medium temperature sizing by adding sodium hydroxide into starch, and then further mixing with synthetic polyacrylate size, mainly using alkali could promote the dissolution of starch in water (Guo et al. 2010). However, alkali could affect the structure of the polyacrylate and weaken the sizing property when starch was the main size. In our previous work, a new sizing way at medium temperature of $65^{\circ} \mathrm{C}$ known as part-gelatinization sizing technology (PGST) was proposed based on the principle that starch presents a state of irreversible water absorption and high expansion at $55^{\sim} 65^{\circ} \mathrm{C}$ (Wu et al. 2014). Unfortunately, the PGST technology must be equipped with a gelatinization device to make a part starch be pregelatinized. Subsequently, we explored the feasibility of sizing on the basis of quaternary ammonium cationic starch for cotton yarn and acetic anhydride esterified starch for PLA filament at $65^{\circ} \mathrm{C}$ under wet conditions (Yang et al. 2017; Shen et al. 2017). In addition, we also achieved starch gelatinization at $60-65^{\circ} \mathrm{C}$ by the synergistic effect of ethanol-alkali system on starch, and showed the same gelatinization characteristics as the raw starch at $100{ }^{\circ} \mathrm{C}$ (Deng et al. 2017). Although there were also report on the introduction of hydrophilic groups into starch structure through hydroxypropylation to improve their solubility and paste clarity 
(Fouladi and Mohammadi 2014; Li et al. 2018b), it was difficult to obtain high molar substitution (MS) for hydroxypropylated starch (HPS), which was mainly due to the reaction staying on the granule surface, resulting in its limited solubility in water at medium temperature. Meanwhile, in addition to the use of the sophisticated pretreatment, repeated filtration, drying, and crushing process, the above methods also increased the production cost of size, leading to a new pollution.

In this work, we will use acid to etch starch to generate channels in starch granules, which will provide space for the full contact between propylene oxide and starch chains within the granule. Meanwhile, acid can also effectively reduce the molecular weight of starch to expose more active groups and promote the reaction between hydroxyl groups and propylene oxide. It is expected to obtain HPS with high molar substitution and realize water solubility at medium temperature. In addition, different from the previous method of preparing medium-temperature water-soluble starch under wet condition, we report a low-energy, simple and efficient method to prepare the water-soluble starch at medium temperature and its structure and property. Based on the characteristic of easy ring-opening reaction from epoxy group in alkaline condition, a white HPS emulsion (about 40\% content) that can be used directly for warp sizing was obtained by chemical bonding the propylene oxide on the active hydroxyl group at $\mathrm{C}_{2}, \mathrm{C}_{3}$, and $\mathrm{C}_{6}$ positions from anhydroglucose unit of starch, and further simple dialyzing against deionized water to the the reacting mixture. The bonding position and reaction rule of hydroxypropyl group on the active hydroxyl group from anhydroglucose unit of starch were studied by Fourier transform infrared spectrometer (FTIR) and nuclear magnetic resonance spectroscopy ( ${ }^{1} \mathrm{H}$ NMR), and the gelatinization mechanism of the formed HPS at medium temperature was revealed by X-ray diffraction (XRD), differential scanning calorimetry (DSC), Ultraviolet-visible spectrophotometer (UV-vis), scanning electron microscopy (SEM), and transmission electron microscope (TEM). The storage stability and dilution stability of the medium-temperature water-soluble HPS were investigated, and the mechanical property of its corresponding film was also evaluated. 


\section{Experimental}

Materials

Corn starch (87\% content) was obtained from Shanfeng Starch Co. Ltd. (Shaanxi, China). Epoxy propane (PO, $0.83 \mathrm{~g} / \mathrm{mL}$ ) and Propylene glycol were purchased from Damao Chemical Reagent Ltd. (Tianjin, China). Sodium hydroxide $(\mathrm{NaOH})$, hydrochloric acid $(\mathrm{HCl}, 12 \mathrm{~mol} / \mathrm{L})$ and sulfuric acid $\left(\mathrm{H}_{2} \mathrm{SO}_{4}, 18.4 \mathrm{~mol} / \mathrm{L}\right)$ were provided by Hongyan Chemical Reagents Ltd. (Tianjin, China). Ninhydrin was supplied by Shanghai Aladdin Biochemical Technology Co., Ltd. (Shanghai, China). All reagents were used without further purification.

Synthesis of water-soluble hydroxypropyl starch (HPS) at medium-temperature

The water-soluble HPS was prepared as follows: Firstly, $100 \mathrm{~g}$ of corn starch aqueous suspension with $40 \mathrm{wt} \%$ in concentration was prepared by dispersing starch powders into the deionized water in a water bath at $40^{\circ} \mathrm{C}$ for $30 \mathrm{~min}$, then the dispersion system was adjusted to $\mathrm{pH}=1 \sim 2$ with $\mathrm{HCl}$ solution $(37 \% w / w)$ and the mixture was stirred for $80 \mathrm{~min}$ to reduce the molecular weight of corn starch. Subsequently, the acidified starch system was adjusted to $\mathrm{pH}=11 \sim 12$ with $\mathrm{NaOH}$ solution $(5 \% w / w)$, followed by adding an appropriate amount of epoxy propane (the molar ratio of epoxy propane to anhydroglucose unit of starch is $3: 1 \sim 7: 1$ ) dropwisely in the above starch dispersion system with stirring for $20 \mathrm{~h}$ continuously. After the completion of reaction, the $\mathrm{pH}$ of reacting mixture system was adjusted to neutral with $\mathrm{HCl}$ solution $(5 \% w / v)$, and the reacting mixture was transferred to a dialysis membrane with 3000 Da molecular weight cut off (MWCO) for 4 days in deionized water (replaced water once every $6 \mathrm{~h}$ ) to remove any unreacted epoxy propane and salt with low molecular weight. Finally, the water-soluble HPS emulsion (about 40 wt $\%$ content) with different molar substitution (MS) was obtained from white suspension in dialysis membrane. All the HPS samples were coded in the form of HPS-P3, HPS-P4, HPS-P5, HPS-P6, HPS-P7, where "Pn" means the added amount of 
PO (the molar ratio of epoxy propane to anhydroglucose unit of starch is $n: 1$ ).

Determining the molar substitution $(M S)$ and reaction efficiency of water-soluble HPS

The MS for water-soluble HPS was measured using a UV-visible spectrophotometer (Lambda 950, Perkin Elmer, USA). The method included hydrolysis of hydroxypropyl group to propylene glycol, then propylene glycol was dehydrated to propionaldehyde and allyl alcohol, and these products were reacted with ninhydrin to produce purple. Firstly, the absorbance of propylene glycol with different concentrations $(10 \sim 50 \mu \mathrm{g} / \mathrm{ml})$ at $\lambda=590 \mathrm{~nm}$ was measured by UV-vis spectrophotometer, and its standard curve was established. Eventually, the $M S$ of water-soluble HPS samples was calculated according to the standard curve. The specific operation method was as follows (Chen et al. 2021): $1 \mathrm{~g}$ of propylene glycol solution was added into a $1000 \mathrm{~mL}$ volumetric flask, then diluted with distilled water to the specified scale. Subsequently, 1, 2, 3, 4, and $5 \mathrm{~mL}$ of the above solution was added into five $100 \mathrm{~mL}$ volumetric flask, respectively, and diluted with distilled water to the specified scale, to obtain series of standard solutions with propylene glycol content of $10,20,30,40,50 \mu \mathrm{g} / \mathrm{ml}$, respectively. $1 \mathrm{~mL}$ of the above five kinds of propylene glycol solutions were added into a $25 \mathrm{~mL}$ colorimetric tube, respectively, then the tubes were inserted into the water, $8 \mathrm{~mL}$ of concentrated sulfuric acid was slowly added the above tubes along the wall, and the tubes was tightened. Subsequently, the tubes were moved to a boiling water bath for $3 \mathrm{~min}$, and immediately placed them in ice bath to cool the samples to $5^{\circ} \mathrm{C}$. $0.6 \mathrm{~mL}$ of $3 \%$ ninhydrin hydrate was added into the above tubes and shaken evenly, and put them into $25^{\circ} \mathrm{C}$ water bath for $100 \mathrm{~min}$, make them develop color. Finally, the tubes were fixed the the volume to $25 \mathrm{~mL}$ using concentrated sulfuric acid, stand for $5 \mathrm{~min}$, and the absorbance of purple solution was measured by UV-vis spectrophotometer at $\lambda=$ $590 \mathrm{~nm}$ to determine the standard curve of propylene glycol.

$0.07 \mathrm{~g}$ of HPS sample was added into $100 \mathrm{~mL}$ volumetric flask, and then put 25 $\mathrm{mL}$ of $0.5 \mathrm{~mol} / \mathrm{L}$ concentrated sulfuric acid into the above volumetric flask. 
Subsequently, the volumetric flask was put into $100^{\circ} \mathrm{C}$ water bath until the sample was completely dissolved. After the sample was cooled to room temperature, diluted it with distilled water to the specified scale. $1 \mathrm{~mL}$ of the above solution was added into a $25 \mathrm{~mL}$ colorimetric tube, and the next operation method was the same as above. Finally, the content of propylene glycol in HPS samples was calculated according the standard curve. The measured content $(\mathrm{g})$ of propylene glycol $\left(W_{P G}\right)$ in $100 \mathrm{~g}$ HPS is converted into hydroxypropyl equivalent using the following equation.

$$
M S=\frac{2.84 W_{P G}}{100-W_{P G}}
$$

The reaction efficiency $(R E)$ of HPS refers to the percentage of propylene oxide (PO) participating in the etherification reaction, which is calculated as the following formula.

$$
R E=\frac{M S}{\text { The molar ratio of etherifying agent PO to starch }} \times 100
$$

Determining the solubility of water-soluble HPS

$100 \mathrm{~g}$ of HPS emulsion with $1 \mathrm{wt} \%$ in concentration was prepared by diluting previous obtained HPS emulsion with $40 \mathrm{wt} \%$ content with deionized water. Then the diluted HPS emulsion was stirred immediately at $65{ }^{\circ} \mathrm{C}$ in a water bath for $1 \mathrm{~h}$, followed by centrifuge treatment for $20 \mathrm{~min}$ with a force of $12 \mathrm{kN}$. The all upper layer was cast onto a polyester plastic plate $(25 \mathrm{~cm} \times 15 \mathrm{~cm})$ to form a film under the natural state, followed by peeled off the film carefully, and dried in an oven at $40{ }^{\circ} \mathrm{C}$ to determine the film weight until its weight was constant. The solubility $(S)$ of water-soluble HPS was calculated by the following formula (Chun et al. 2016):

$$
S=\frac{B}{A} \times 100
$$

Where $A$ is the total dry weight of HPS contained in the original centrifuge tube, $g ; B$ is the dry weight of HPS film after drying, $g$.

In addition, the solubility effect of water-soluble HPS could be presented indirectly by their light transmittance (Craig et al. 1989). We prepared the HPS 
solution ( $1 \%$ content) with different $M S$ at $60{ }^{\circ} \mathrm{C}, 65^{\circ} \mathrm{C}$ and $95{ }^{\circ} \mathrm{C}$, respectively, and then the light transmittance (T) above $500 \mathrm{~nm}$ of the sample solution was measured by UV-vis spectrophotometer (Lambda 950, Perkin Elmer, USA), as basis for evaluation of solubility effect.

\section{Preparation of HPS film}

The HPS film was prepared by a tape-casting method. The pre-prepared $40 \%$ HPS emulsion was diluted to $3 \%$ with deionized water and heated it to $65^{\circ} \mathrm{C}$ for $1 \mathrm{~h}$ under mechanical stirring. Then, the paste was cast onto a polyester plastic plate and evaporating water under the natural state, followed by demoulding and stored in a desiccator $\left(65 \pm 2 \% \mathrm{RH}, 20 \pm 1^{\circ} \mathrm{C}\right)$ prior to the measurement.

\section{Characterization}

\section{FTIR analysis}

The water-soluble HPS emulsion was dried and ground into powder, then mixed it with $\mathrm{KBr}$ and pressed into a thin slice, which was immediately tested using a FTIR spectrometer (Spotlight 400, Parkin Elgen, UK) in the wavelength range from 4000 to $500 \mathrm{~cm}^{-1}$.

\section{${ }^{1} H$ NMR analysis}

The ${ }^{1} \mathrm{H}$ NMR spectra measurement of the sample was carried out with an NMR spectrometer (Bruker AV III, 400 M, Germany). The deuterated dimethylsulfoxide (DMSO- $\mathrm{d}_{6}$ ) was used as the solvent, and the trimethylsilane (TMS) was used as internal standard.

\section{XRD analysis}

XRD instrument (Dmax-rapid II, Japan) with $\mathrm{Cu}-\mathrm{K} \alpha$ radiation at a voltage of 30 $\mathrm{kV}$ and current of $15 \mathrm{~mA}$ was used to test the crystallinity of the sample. The scanning speed of $10^{\circ} / \mathrm{min}$, the step width of $0.02^{\circ}$, and the scanning range of $5^{\sim} 30^{\circ}$. The 
crystallinity $\left(X_{c}\right)$ of the samples were calculated by the following equation:

$$
X_{c}=\frac{A_{c}}{A_{c}+A_{a}} \times 100 \%
$$

Where $A_{c}$ and $A_{a}$ are the integrated areas of all crystalline peaks and the amorphous halo, respectively.

Morphological analysis

The freeze-dried HPS samples were sprayed gold, and the granule surface morphology was observed by scanning electron microscope (SEM) (Quanta-450-PEG, PEI, USA). The morphology of water-soluble HPS solution was observed by a transmission electron microscopy (TEM) (JEM-F200, Japan). The sample solution was dripped onto the copper grid and air-dried, followed by staining it with phosphotungstic acid $(2 \%, \mathrm{w} / \mathrm{w})$ for $3 \mathrm{~min}$ and observed its morphology.

\section{DSC analysis}

The gelatinization behaviour of the sample was analysed using a DSC analyzer (STA449 F3, Netzsch, Germany). About $30 \mu \mathrm{L}$ starch suspension $(30 \%$, w/w) was used for each test under $\mathrm{N}_{2}$ atmosphere, heated in a $40 \mu \mathrm{L} \mathrm{Al}{ }_{2} \mathrm{O}_{3}$ crucible to $20-80{ }^{\circ} \mathrm{C}$ with the rate of $10^{\circ} \mathrm{C} / \mathrm{min}$.

\section{Mechanical property of film}

The mechanical properties of the HPS films were measured on a HD021N electronic single yarn strength tester (Nantong Hongda Instrument Co., Ltd, China) at a speed of $50 \mathrm{~mm} / \mathrm{min}$ according to ASTM/D2256-2015. Films of $120 \mathrm{~mm} \times 5 \mathrm{~mm}$ (analyzed area $=100 \mathrm{~mm} \times 5 \mathrm{~mm}$ ) were used to test the elongation at break. The flex resistance was estimated according to the previous literature (Li et al. 2016). The data reported were averages of 10 measurements. The average thickness of films was 0.06 $\mathrm{mm}$. 


\section{Results and discussion}

245 Fabrication of water-soluble HPS and its corresponding morphology

The water-soluble HPS are obtained by a two-step method as illustrated in Fig. 1a. Firstly, the corn starch was pretreated with acid hydrolysis to reduce the molecular weight of starch macromolecules, then the acidified starch was reacted with epoxy propane to form HPS under alkaline conditions. Fig. 1b shows the FTIR spectra of raw starch and HPS with different amounts of propylene oxide (PO). For the spectrum of raw starch, the peaks at $3400,2930,1425$ and $850 \mathrm{~cm}^{-1}$ are attributed to $\mathrm{O}-\mathrm{H}, \mathrm{C}-\mathrm{H}$, $-\mathrm{CH}_{2}$ stretching vibration and $\mathrm{C}-\mathrm{H}$ anti-stretching vibration, respectively (Hay et al. 2018; Zhang et al. 2013). Moreover, the absorption peak at $1024 \mathrm{~cm}^{-1}$ is belonged to the characteristic peak of $\alpha-1,4$ and $\alpha-1,6$ glucosidic bond, as well as six-member heterocyclic containing carbon and oxygen (C-O-C) (Woggum et al. 2015). Compared with the FTIR spectrum of raw starch, in addition to the characteristic peaks of starch appeared on the HPS structure, the intensities of the peaks for the C-O-C $\left(1024 \mathrm{~cm}^{-1}\right)$, C-H $\left(2930 \mathrm{~cm}^{-1}\right)$ and $-\mathrm{CH}_{2}\left(1425 \mathrm{~cm}^{-1}\right)$ groups increase slightly due to the introduction of epoxy propane can provide abundant C-O-C, C-H and $-\mathrm{CH}_{2}$ groups (Yao et al. 2019). From the result of FTIR, the hydroxypropyl group is successfully conjugated to the backbone of starch.

To further explore the the bond position and reaction rule of hydroxypropyl on shown in Fig. 1c. It can be seen that the signal peaks at $\delta=3.2-5.7$ ppm were ascribed to the resonance protons in the AGU units of starch, and the methylene and methine non-anomeric proton peaks in starch could be observed at $\delta=3.2-4.1 \mathrm{ppm}$ (Zhao et al. 2015). Meanwhile, the signal peaks at $\delta=5.67,5.52$ and $5.40 \mathrm{ppm}$ were belonged to $\mathrm{C}_{2}, \mathrm{C}_{3}$ and $\mathrm{C}_{6}$ protons of residual hydroxyl in the $\mathrm{AGU}$ units of starch, respectively (Zhao et al. 2015; Xu and Seib 1997). In comparison with the raw starch, in addition to the characteristic signal proton peaks of starch were occurred on the ${ }^{1} \mathrm{H}$ NMR 
spectra of HPS, the new doublet signal peak at $\delta=1.18 \mathrm{ppm}$ assigned to the terminal methyl of the hydroxypropyl groups was observed, especially the proton peak at $\delta=$ $1.18 \mathrm{ppm}$ was more prominent with the increase of PO, indicating that hydroxypropyl was introduced onto the starch backbone (Zhao et al. 2015). Moreover, it can be seen that the integral area for the signal at $\delta=5.67 \mathrm{ppm}$ assigned to the $\mathrm{C}_{2}$ proton of residual hydroxyl in HPS was lower than that of the raw starch, followed by the $\mathrm{C}_{6}(\delta$ $=5.40 \mathrm{ppm})$ and $\mathrm{C}_{3}(\delta=5.52 \mathrm{ppm})$ protons of residual hydroxyl, respectively. This is caused by the $\mathrm{C}_{2}$ hydroxyl exhibits a relatively high acidity due to its proximity to the anomeric centre (Tuschhoff 1986). It means that hydroxypropylation mainly occurred on the hydroxyl group of $\mathrm{C}_{2}$ position in the AGU unit of starch, and the substitution level at $\mathrm{C}_{6}$ was slightly higher than that at $\mathrm{C}_{3}$. Therefore, the reaction activity of hydroxypropyl on the $-\mathrm{OH}$ from $\mathrm{AGU}$ units of starch followed the rule: $\mathrm{C}_{2}>\mathrm{C}_{6}>\mathrm{C}_{3}$. Zhao et al also found the same rule in study on the level and position of substituents in cross-linked and hydroxypropylated sweet potato starches (Zhao et al. 2015).

(a)

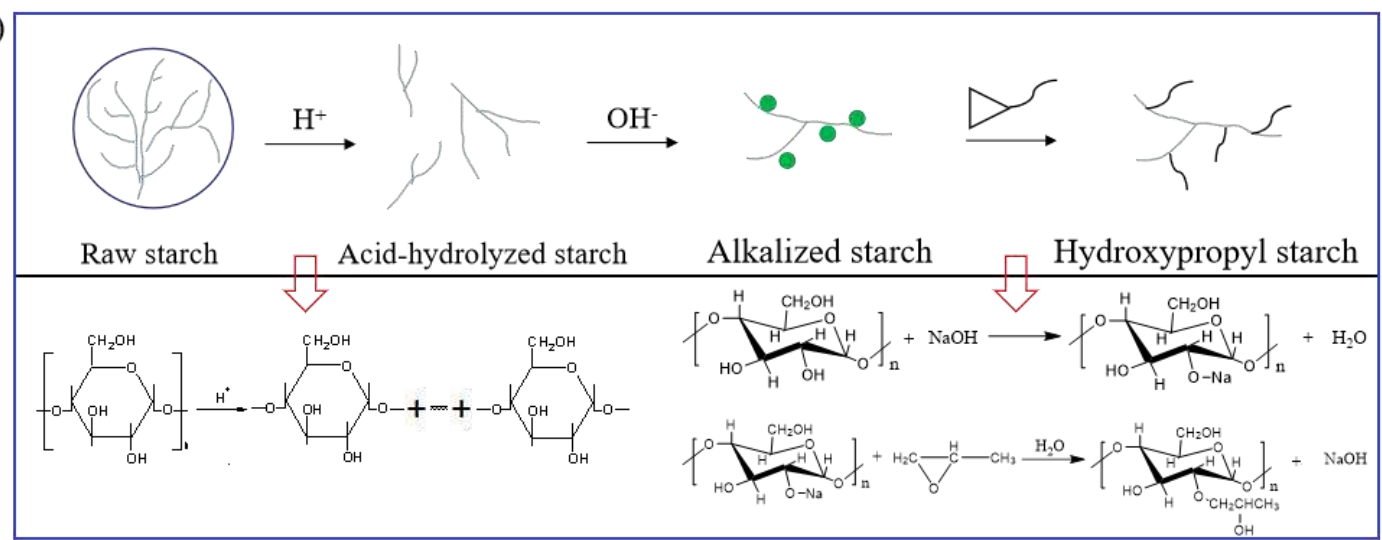

(b)

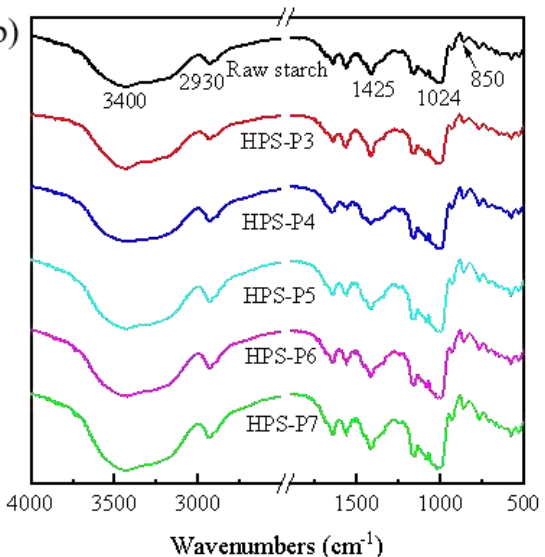

(c)

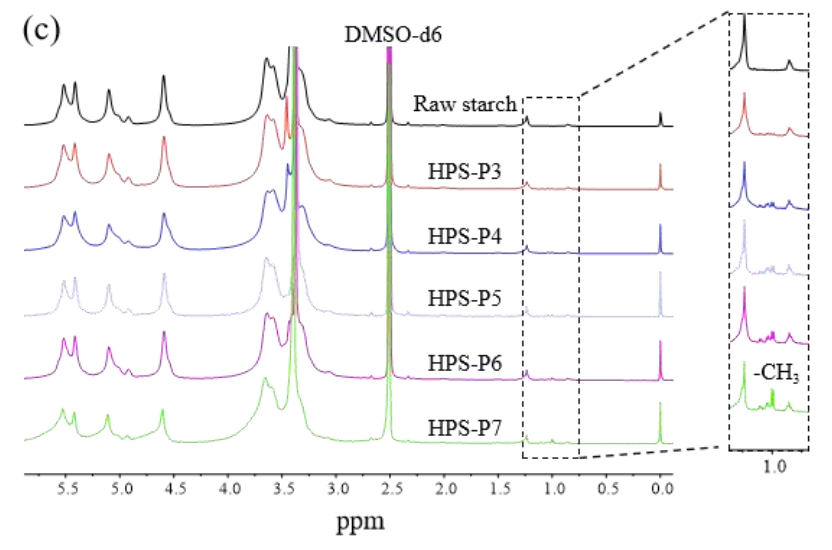

Fig.1. (a) Preparation of the water-soluble HPS; (b) FTIR and (c) ${ }^{1} \mathrm{H}$ NMR spectra of the raw starch and HPS with different amounts of PO. 

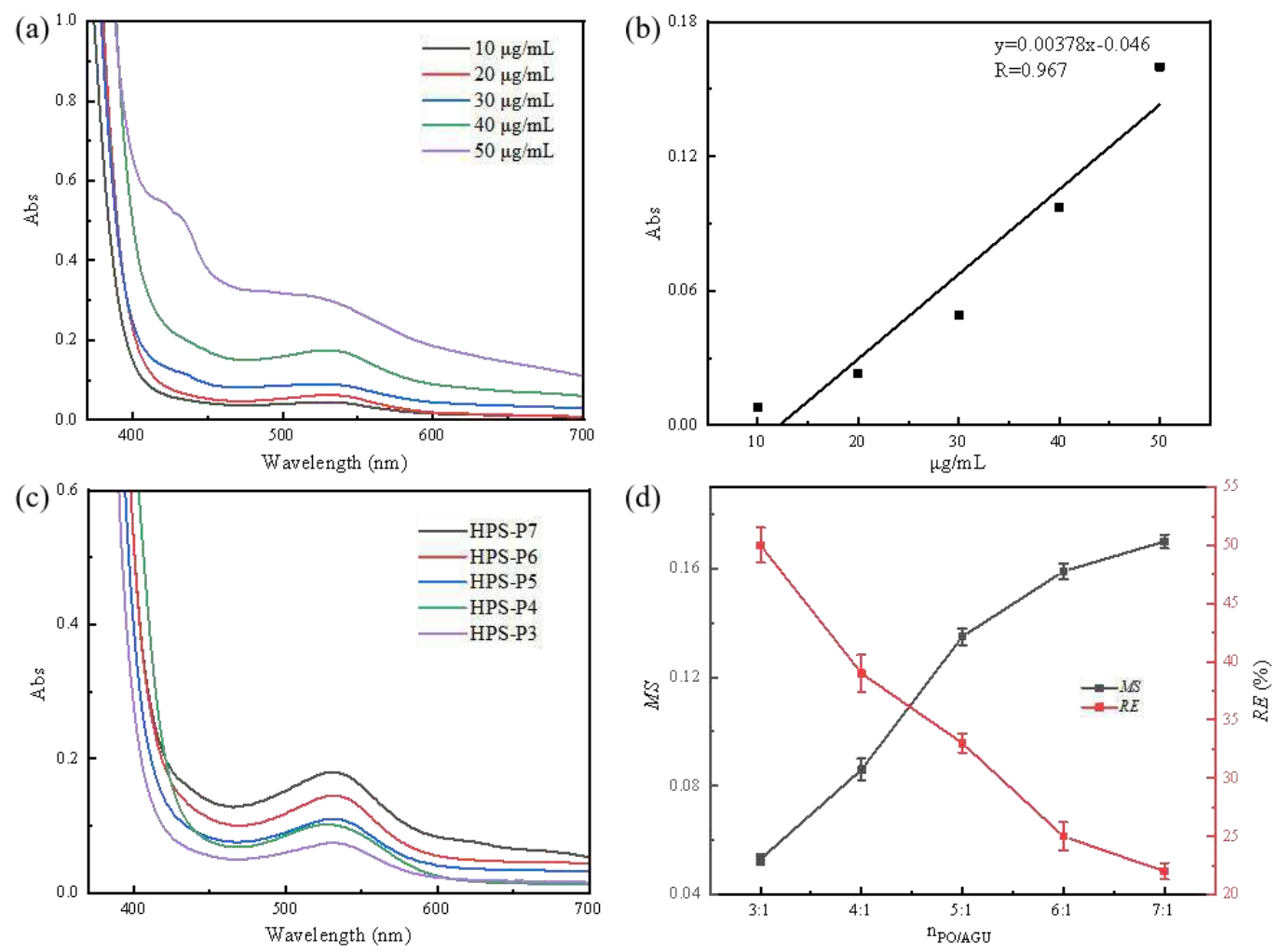

Fig.2 (a) UV-vis spectra of propylene glycol with different concentrations $(10 \sim 50 \mu \mathrm{g} / \mathrm{mL})$; (b)

Calibration curve of propylene glycol; (c) UV-vis spectra of HPS samples; (d) The effect of the molar ratio of PO to anhydroglucose unit of starch on $M S$ and the reaction efficiency $(R E)$ of HPS.

The $M S$ for HPS was calculated by UV-vis spectrum, as shown in Fig.2. Fig.2a shows the UV-vis spectra of propylene glycol with different concentrations (10 50 $\mu \mathrm{g} / \mathrm{mL})$. It can be seen that the absorbance of propylene glycol increase with the increase of its concentration, and its concentration and absorbance exhibit a good linear correlation, the correlation coefficient is 0.967 (Fig. 2b). Fig.2c shows the UV-vis spectra of HPS with different amounts of PO. For the HPS samples, a higher PO content led to greater absorbance. According to the standard curve of propylene glycol, the $M S$ results and the reaction efficiency $(R E)$ of HPS with the different molar ratio of $\mathrm{PO}$ to $\mathrm{AGU}$ of starch were obtained, as shown in Fig.2d. It was obvious that the $M S$ of HPS increased from 0.053 to 0.170 when the molar ratio of PO to AGU of starch varied from 3:1 to 7:1, which means that the $M S$ of synthesized HPS could be easily adjusted by changing the addition amount of PO. But the $R E$ of hydroxypropyl gradually decreased from $50 \%$ to $22 \%$ with the increasing of PO content in the AGU 
of starch. This may be attributed to the concentration of reagents participating in the reaction system increases with the increasing of PO, leading to greater contact probability between $\mathrm{PO}$ and $-\mathrm{OH}$ of starch glucose ring, and the $M S$ was increased. But as the substitution degree of hydroxyl groups on the AGU units of starch increased, the bonding space of hydroxypropyl group on the structure of starch became smaller, resulting in the decrease of $R E$ with the increasing of PO.

Fig. 3 showed the SEM surface morphology of raw starch, acid hydrolyzed starch, $\mathrm{HPS}_{M S}=0.053, \mathrm{HPS}_{M S}=0.135$, and $\mathrm{HPS}_{M S}=0.170$ granules. The raw starch granule exhibited smooth appearance (Fig.3a). However, for acid hydrolyzed starch granule, obvious expansion and groove could be observed on its surface (Fig.3b). Furthermore, it can be seen that there were channels in the acid hydrolyzed starch granule from the enlarged view, which provides reactivity for the PO to penetrate into starch chains within the granule. For HPS granules, their overall structure were loose, especially in HPS $_{M S=0.170}$ granule with high $M S$ (Fig.3c-e). This will be beneficial to the directional adsorption and diffusion of water molecules in starch microstructure.
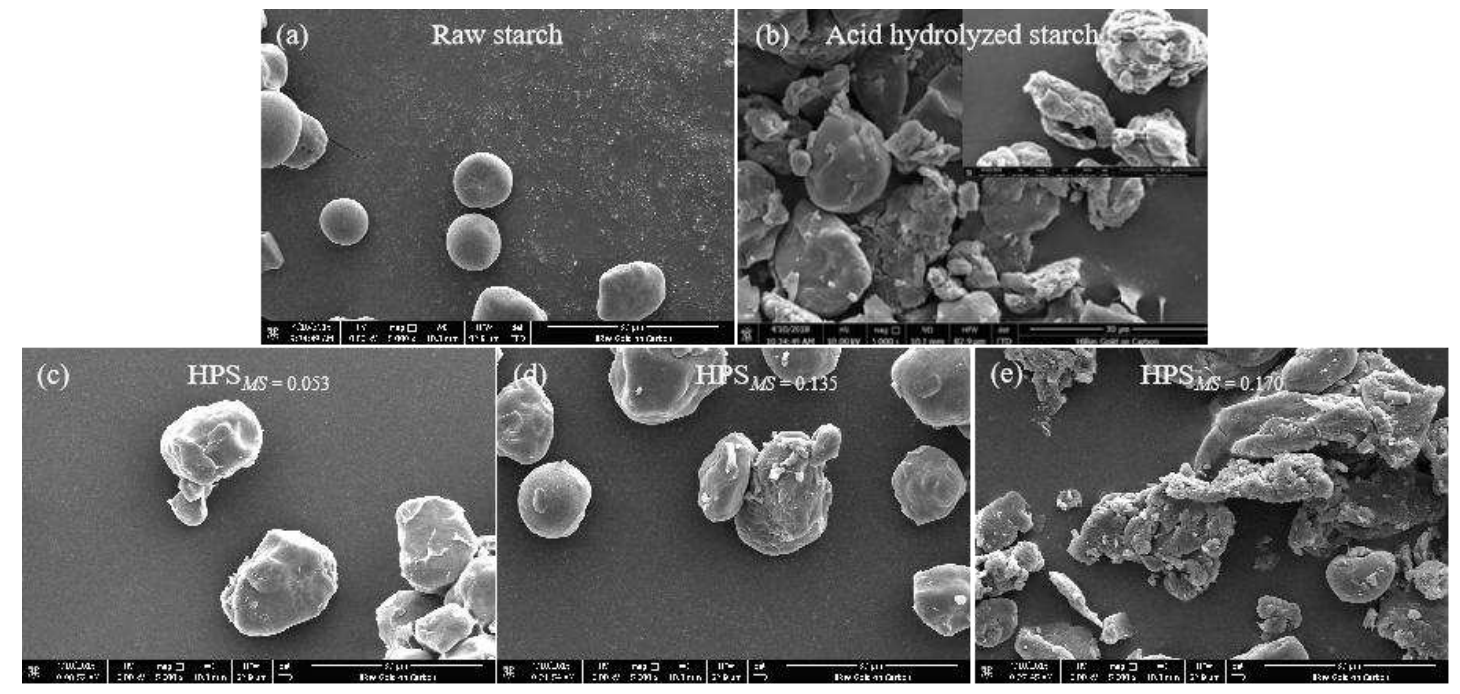

Fig.3 SEM images of the raw starch, acid hydrolyzed starch and HPS with different MS.

Gelatinization temperature and solution stability of water-soluble HPS

The gelatinization temperature of water-soluble HPS was investigated by XRD, DSC, TEM and UV-Vis. The XRD patterns of raw starch and HPS were shown in Fig. 4a. For both the raw starch and HPS, the four obvious diffraction peaks at $2 \theta=15.0^{\circ}$, 
$17.0^{\circ}, 17.9^{\circ}$, and $22.9^{\circ}$ were presented, but the intensities of crystalline peak for HPS with different $M S$ was lower than that of raw starch, indicating that the original crystalline structure of starch had been disrupted after hydroxypropyl modification, and the crystallinity of raw starch and $\operatorname{HPS}_{M S=0.170}$ was $52.41 \%$ and $29.4 \%$, respectively. The reduction of crystallinity could further improve the adsorption ability of HPS to water molecules (Wang et al. 2019).

Fig. $4 \mathrm{~b}$ shows the DSC thermograms of the raw starch and HPS samples, with associated gelatinization parameters shown in Table 1. Compared with the DSC thermogram of raw starch, the gelatinization onset $\left(T_{0}\right)$, peak gelatinization temperature $\left(T_{\mathrm{p}}\right)$ and conclusion temperatures $\left(T_{\mathrm{c}}\right)$ as well as enthalpy change $(\Delta H)$ of HPS samples decreased with $M S$ increased from 0.053 to 0.170 . This meant that hydroxypropyl modification could reduce the gelatinization temperature of starch. In this regard, the introduction of hydroxypropyl groups could weaken the hydrogen bond in the helix structure of starch molecular chain, and make it easier for starch granules to undergo swelling and melting of crystallites, thus reducing the gelatinization temperature and enthalpy change (Woggum et al., 2015). Based on the fact, the solubility and light transmittance of HPS with $M S$ of 0.053 , 0.135 and 0.170 at the $60{ }^{\circ} \mathrm{C}, 65^{\circ} \mathrm{C}$ and $95^{\circ} \mathrm{C}$ were evaluated, respectively, as shown in Fig. 4d. The solubility and light transmittance of HPS with different $M S$ increased with the increase of temperature. Especially, both the solubility and transmittance of $\operatorname{HPS}_{M S=0.135}$ and $\operatorname{HPS}_{M S=0.170}$ were more than $90 \%$ and $55 \%$ at $65{ }^{\circ} \mathrm{C}$, respectively, showing the similar dissolution state as that of $95^{\circ} \mathrm{C}$. This result showed HPS with good water solubility at $65{ }^{\circ} \mathrm{C}$ could be obtained by adjusting the molar ratio of epoxy propane to anhydroglucose unit of starch was not less than 5:1 and as long as $M S \geqslant$ 0.1. Moreover, from the TEM photographs of $\mathrm{HPS}_{M S=0.170}$ dissolved in water (Fig. 4c), the dissolution state for $\mathrm{HPS}_{M S=0.170}$ dissolved at $65{ }^{\circ} \mathrm{C}$ was similar to that of at $95{ }^{\circ} \mathrm{C}$, and both of them showed uniform spherical morphology, which further confirmed that the HPS obtained in this work can be completely dissolved at $65^{\circ} \mathrm{C}$.

In order to explore the stability of the medium temperature water-soluble HPS, both the storage and dilution stability of $\mathrm{HPS}_{M S=0.170}$ dissolved at $65^{\circ} \mathrm{C}$ and $95^{\circ} \mathrm{C}$ were 
further analyzed, respectively, as shown in Fig. 4e. There were no obvious difference

358 in the solution appearance for $\operatorname{HPS}_{M S}=0.170$ dissolved at $65^{\circ} \mathrm{C}$ and $95^{\circ} \mathrm{C}$, exhibiting a

359 uniform distribution and translucent state, as well as no sediment and

360 layering phenomenon for the water-soluble $\operatorname{HPS}_{M S}=0.170$ (dissolved at $65^{\circ} \mathrm{C}$ ) at

361 concentration of $10 \%$ and diluted to $1 \%$ was observed on the bottom of the bottle after

362 standing for $48 \mathrm{~h}$, indicating that the obtained medium temperature water-soluble HPS

363 had high storage stability and dilution stability.

(a)

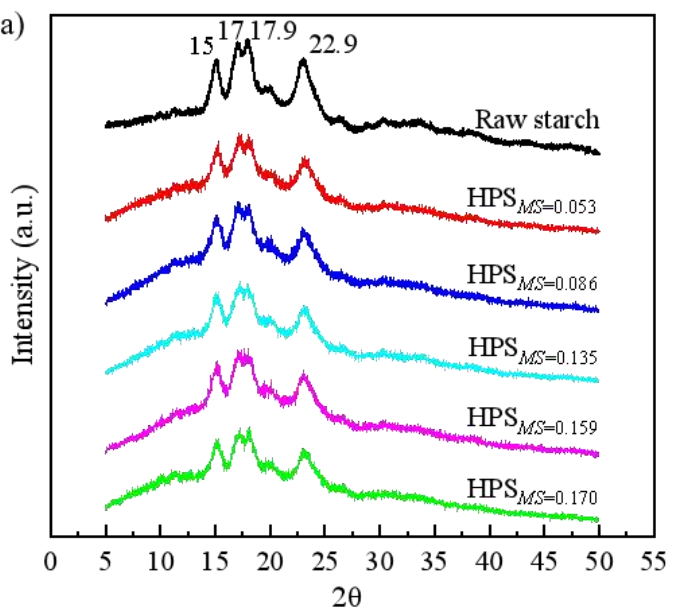

(c)

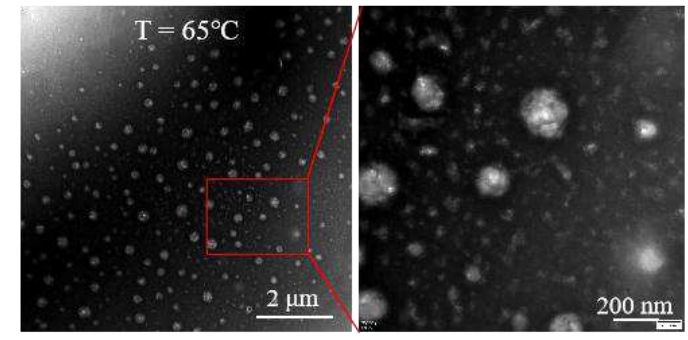

(b)

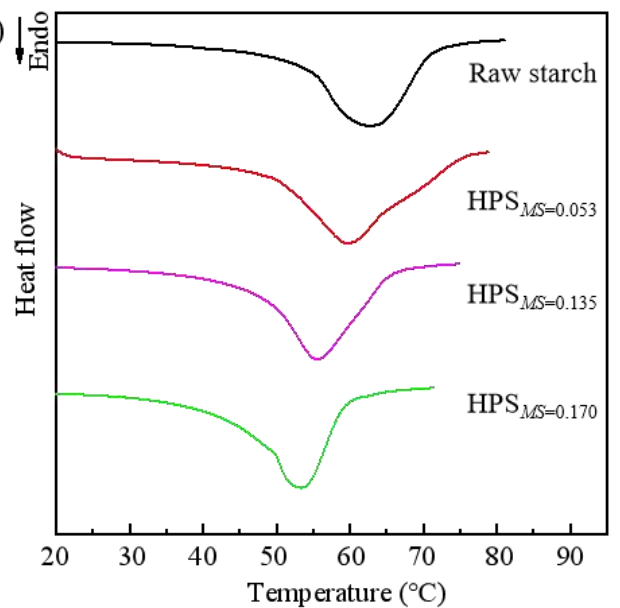

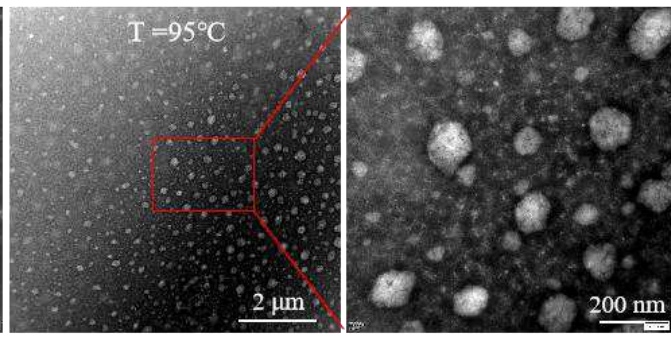

(e)
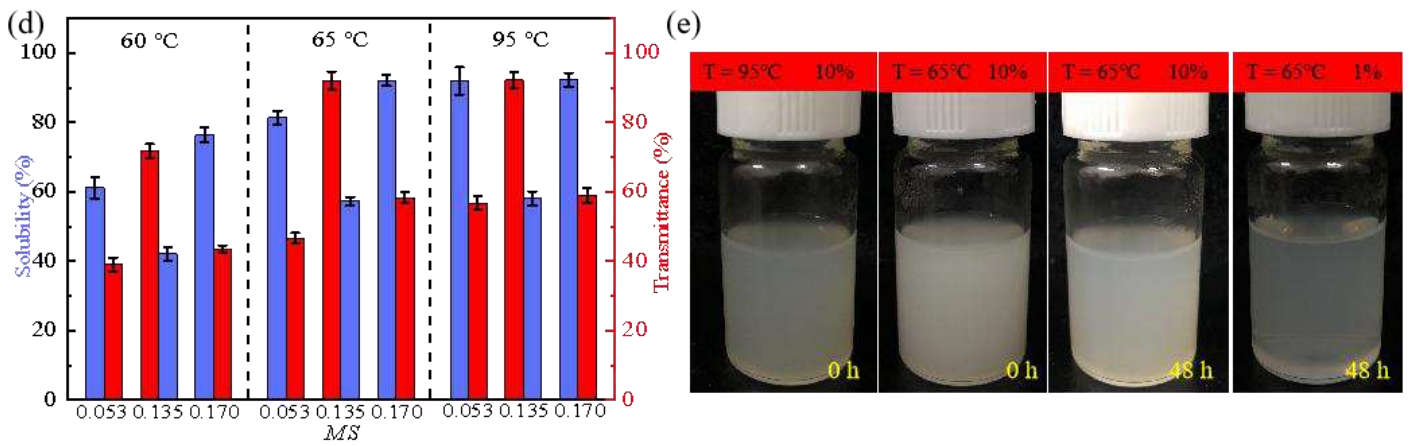

Fig.4. (a) XRD patterns of the raw starch and HPS with different MS; (b) DSC spectra of the raw starch and HPS with different $M S$; (c) TEM photographs of $\mathrm{HPS}_{M S=0.170}$ dissolved at $65{ }^{\circ} \mathrm{C}$ and $95^{\circ} \mathrm{C}$; (d) Comparison of solubility and transmittance of HPS with different $M S$ at $60{ }^{\circ} \mathrm{C}, 65{ }^{\circ} \mathrm{C}$, and $95^{\circ} \mathrm{C}$, respectively; (e) Storage and dilution stability of $\mathrm{HPS}_{M S}=0.170$ dissolved at $65^{\circ} \mathrm{C}$ and $95^{\circ} \mathrm{C}$, respectively. 
Table 1 Onset temperature $\left(T_{0}\right)$, peak gelatinization temperature $\left(T_{\mathrm{p}}\right)$, conclusion temperature $\left(T_{\mathrm{c}}\right)$ and enthalpy change $(\Delta H)$ of gelatinization measured by DSC for native and water-soluble HPS samples.

\begin{tabular}{lllll}
\hline Sample & $T_{0}\left({ }^{\circ} \mathrm{C}\right)$ & $T_{\mathrm{p}}\left({ }^{\circ} \mathrm{C}\right)$ & $T_{\mathrm{c}}\left({ }^{\circ} \mathrm{C}\right)$ & $\Delta H(\mathrm{~J} / \mathrm{g})$ \\
\hline Raw starch & $53.4 \pm 0.2$ & $62.5 \pm 0.3$ & $76.6 \pm 0.4$ & $12.8 \pm 0.3$ \\
$\mathrm{HPS}_{M S=0.053}$ & $51.1 \pm 0.1$ & $60.1 \pm 0.2$ & $75.3 \pm 0.2$ & $10.4 \pm 0.5$ \\
$\mathrm{HPS}_{M S=0.135}$ & $45.6 \pm 0.4$ & $56.3 \pm 0.2$ & $69.5 \pm 0.3$ & $7.8 \pm 0.4$ \\
$\mathrm{HPS}_{M S=0.170}$ & $42.3 \pm 0.4$ & $53.4 \pm 0.3$ & $65.4 \pm 0.2$ & $6.5 \pm 0.6$ \\
\hline
\end{tabular}

Mechanical property of water-soluble HPS film

The relationship between $M S$ of HPS films (obtained by dissolving at $65^{\circ} \mathrm{C}$ ) and the tensile strength, elongation at break and flex resistance under $65 \%$ relative humidity was shown in Fig.5a. With an increase of $M S$, the tensile strength of HPS films decreased, while both the elongation at break and flex resistance increased, which meant that high $M S$ of hydroxypropyl could improve the plasticity of starch films. This could be explained by the inter- and intra-molecular hydrogen bonding interactions of starch was attenuated when hydroxypropyl group was introduced onto starch backbone (Xia et al. 2018), and the stable non-polar alkyl chain with flexible characteristic on starch structure, resulting in dramatically flexibility effect. Moreover, benefiting from the introduction of hydrophilic ether bonds on starch structure, the more water molecules could be combined onto the HPS films compared with that of raw starch film under $65 \%$ relative humidity, which led to obvious plasticizing effect (Zhang et al. 2018). The appearance photographs in Fig. 5b showed the the raw starch film was incomplete clearly, while HPS with $M S$ greater than 0.1 exhibited complete and continuous appearance from macroscopic view, demonstrating the high flexibility of HPS films. 
390 (a)

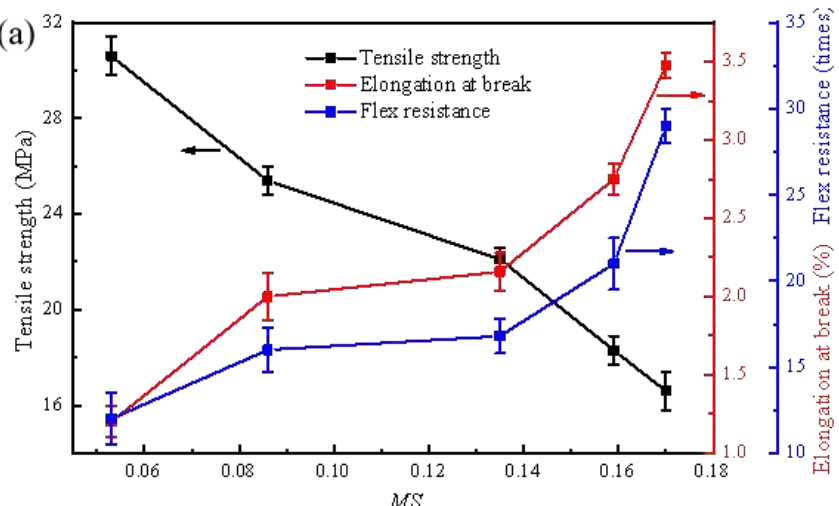

(b)

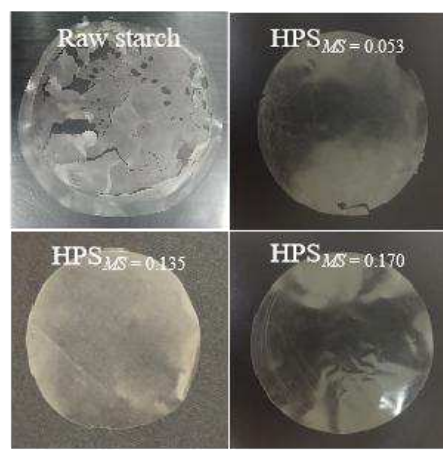

Fig.5. (a) Mechanical properties of HPS films with different MS; (b) Appearance photographs of raw starch and medium temperature water-soluble HPS films.

\section{Conclusion}

A series of HPS with the total molar substitution $(M S)$ values of $0.053 \sim 0.170$ was obtained via a etherification of acidified corn starch with epoxy propane for introducing hydroxypropyl onto the backbones of starch. The water-soluble HPS at $65^{\circ} \mathrm{C}$ with solubility of over $90 \%$ and light transmittance of above $55 \%$ could be obtained when the molar ratio of epoxy propane to anhydroglucose unit of starch was not less than 5:1 (ie. MS of HPS was greater than 0.1). The morphology of starch granule changed obviously, and its crystallinity, gelatinization temperatures $\left(T_{0}, T_{\mathrm{p}}\right.$ and $\left.T_{\mathrm{c}}\right)$ and enthalpy change $(\Delta H)$ decreased significantly after hydroxypropylation, which could promote the adsorption and transmission of water molecules in the starch microstructure. Moreover, the obtained water-soluble HPS at $65{ }^{\circ} \mathrm{C}$ exhibited high storage and dilution stability, and presented a similar dissolution state with that of at $95{ }^{\circ} \mathrm{C}$. Interestingly, hydroxypropylation was conducive to good breaking elongation and flex resistance of HPS films at low relative humidity $(65 \% \mathrm{RH})$.

\section{Acknowledgments}

The authors acknowledge support from the National Key Research and Development Program of China (2017YFB0309100), Scientific Research Program of Shaanxi Province Education Department, and Doctoral Research Program of Xi'an Polytechnic University (107020533). 


\section{Conflict of interest}

413 The authors declare that they have no conflict of interest.

414

\section{References}

Craig S, Maningat C, Seib P, Hoseney R (1989) Starch paste clarity. Cereal Chemistry 66: 173-182.

Chen P, Zhang Y, Qiao Q, Tao X, Liu P, Xie F (2021) Comparison of the structure and properties of hydroxypropylated acid-hydrolysed maize starches with different amylose/amylopectin contents. Food Hydrocolloid 110: 106134.

Chun E, Oh S, Kim H, Kim B, Baik M (2016) Effect of high hydrostatic pressure treatment on conventional hydroxypropylation of maize starch. Carbohyd Polym 146: 328-336.

Deng L, Wang W, Wu H, Shen Y (2017) Application effect research of sizing technology at lower temperature. Cotton Text Tech 45: 10-13.

Djordjevic S, Kovacevic S, Djordjevic D, Konstantinovic S (2019) Sizing process of cotton yarn by size from a copolymer methacrylic acid and hydrolyzed potato starch. Text Res J 89(17):3457-3465.

Fouladi E, Mohammadi N (2014) Effects of acid-hydrolysis and hydroxypropylation on functional properties of sago starch. Int J Biol Macromol 68: 251-257.

Guo L, Dai C, Li C (2010) A method for sizing warp at room temperature: China, CN 20101022 2890.2. 2010-11-24.

Hay W, Fanta G, Peterson S, Thomas A, Utt K, Walsh K, Boddu V, Selling G (2018) Improved hydroxypropyl methylcellulose (HPMC) films through incorporation of amylose-sodium palmitate inclusion complexes. Carbohyd Polym 188: 76-84.

$\mathrm{Hu}$ J, \& Babu KM (2007) Weaving preparation: warping, sizing, drawing-in and tying. Text Asia 38-39: 27-33.

Jeong H, Baek S, Han S, Jang H, Kim S, Lee H (2018) Novel eco-friendly starch paper for use in flexible, transparent, and disposable organic electronics. Adv Funct Mater 28: 1704433.1-1704433.9.

Jiang Q, Chen S, Deng X, Feng Y, Reddy N, Zhu Q, Liu W, Qiu Y (2019) A sustainable low temperature yarn reinforcing process to reduce water and energy consumptions and 
pollution in the textile industry. Clean Prod 210: 646-652.

Kim H, Jane J, Lamsal B (2017) Hydroxypropylation improves film properties of high amylose corn starch. Ind Crop Prod 95: 175-183.

Li L, Hong Y, Gu Z, Cheng L, Li Z, Li C (2018b). Effect of a dual modification by hydroxypropylation and acid hydrolysis on the structure and rheological properties of potato starch. Food Hydrocolloid 77: 825-833.

Li M, Zhu Z, Pan X(2011) Effects of starch acryloylation on the grafting efficiency, adhesion, and film properties of acryloylated starch-g-poly(acrylic acid) for warp sizing. Text Res J 63(11):683-691.

Li W, Xu Z, Wang Z, Xing J (2018a) One-step quaternization/hydroxypropylsulfonation to improve paste stability, adhesion, and film properties of oxidized starch. Polymers 10: $1110-1125$.

Li W, Xu W, Wei A, Xu Z, Zhang C (2016) Quaternization/maleation of cornstarch to improve its adhesion and film properties for warp sizing. Fibers Polym 17: 1589-1597.

Rafikov A, Khakimova M, Fayzullayeva D, Reyimov A (2020) Microstructure, morphology and strength of cotton yarns sized by collagen solution. Cellulose 27: 10369-10384.

Shen Y, Wu H, Xiong R, Mao N (2017) Synthesis and properties of water soluble quaternary ammonium cationic starch sizing materials. J Text Res 38: 73-78.

Tuschhoff, J. V. (1986) Hydroxypropylated starches. In O. B. Wurzburg (Ed.), Modified starches: Properties and uses (pp. 89-95). Boca Raton, FL, USA: CRC Press.

Wang R, Liu P, Cui B, Kang X, Yu B (2019) Effects of different treatment methods on properties of potato starch-lauric acid complex and potato starch-based films. Int J Biol Macromol 124: 34-40.

Wang L, Zhang X, Xu J, Wang Q, Fan X (2020) How starch-g-poly(acrylamide) molecular structure effect sizing properties. Int J Biol Macromol 144(1):403-409.

Woggum T, Sirivongpaisal P, Wittaya T (2015) Characteristics and properties of hydroxypropylated rice starch based biodegradable films. Food Hydrocolloid 50: 54-64.

Wu H, Shen Y, Li D, Mao N, Xu J, Shi B, Chen S, Wu Z (2014) Mechanism of part-gelatinization sizing technology on yarns. J Text Res 35: 47-51.

Wu H, Shen Y, Yao Y, Zhang B (2021) Size formulations for cotton yarn weaving at lower 
relative humidity. Text Res J 91(1-2): 168-174.

Xia H, Kou T, Liu K, Gao Q, Fang G (2018) Recrystallization kinetics of starch microspheres prepared by temperature cycling in aqueous two-phase system. Carbohyd Polym 198: 233-240.

Xu A, \& Seib P A. (1997). Determination of the level and position of substitution in hydroxypropylated starch by high-resolution ${ }^{1} \mathrm{H}-\mathrm{NMR}$ spectroscopy of alpha-limit dextrins. J Cereal Sci 25(1): 17-26.

Xu J, Wang L, Wang J, Fan X, Wang Q, Wang P, Zhang Y, Li C, Yuan J, Yu Y (2016) Partially gelatinized corn starch as a potential environmentally friendly warp-sizing agent. J Clean Prod 112: 3195-3200.

Yang F, Tao L, Li C, Gao L, Yang P (2018) Self-assembled membrane composed of amyloid-like proteins for efficient size-selective molecular separation and dialysis. Nat Commun 9: 5443-5453.

Yang M, Wu H, Shen Y, Yao Y (2017) Sizing of polylactic acid filaments at lower temperature. J Text Res 38: 85-90.

Yao Y, Wang H, Wang R, Chai Y, Ji W (2019) Fabrication and performance characterization of the membrane from self-dispersed gelatin-coupled cellulose microgels. Cellulose 26: $3255-3269$.

Zhang K, Cheng F, Lin Y, Zhou M, Zhu P (2018) Effect of hyperbranched poly(trimellitic glyceride) with different molecular weight on starch plasticization and compatibility with polyester. Carbohyd Polym 195: 107-113.

Zhang L, Wang Y, Liu H, Yu L, Liu X, Chen L, Zhang N (2013) Developing hydroxypropyl methylcellulose/hydroxypropyl starch blends for use as capsule materials. Carbohyd Polym 98(1): 73-79.

Zhao J, Chen Z, Jin Z, Waard P, Buwalda P, Gruppen H, Schols H (2015) Level and position of substituents in cross-linked and hydroxypropylated sweet potato starches using nuclear magnetic resonance spectroscopy. Carbohyd Polym 131: 424-431.

Zhu Z, Wang M, Li W (2015) Starch maleation and sulfosuccinylation to alleviate the intrinsic drawback of brittleness of cornstarch film for warp sizing. Fibers Polym 16: 1890-1897. 
Figures

(a)

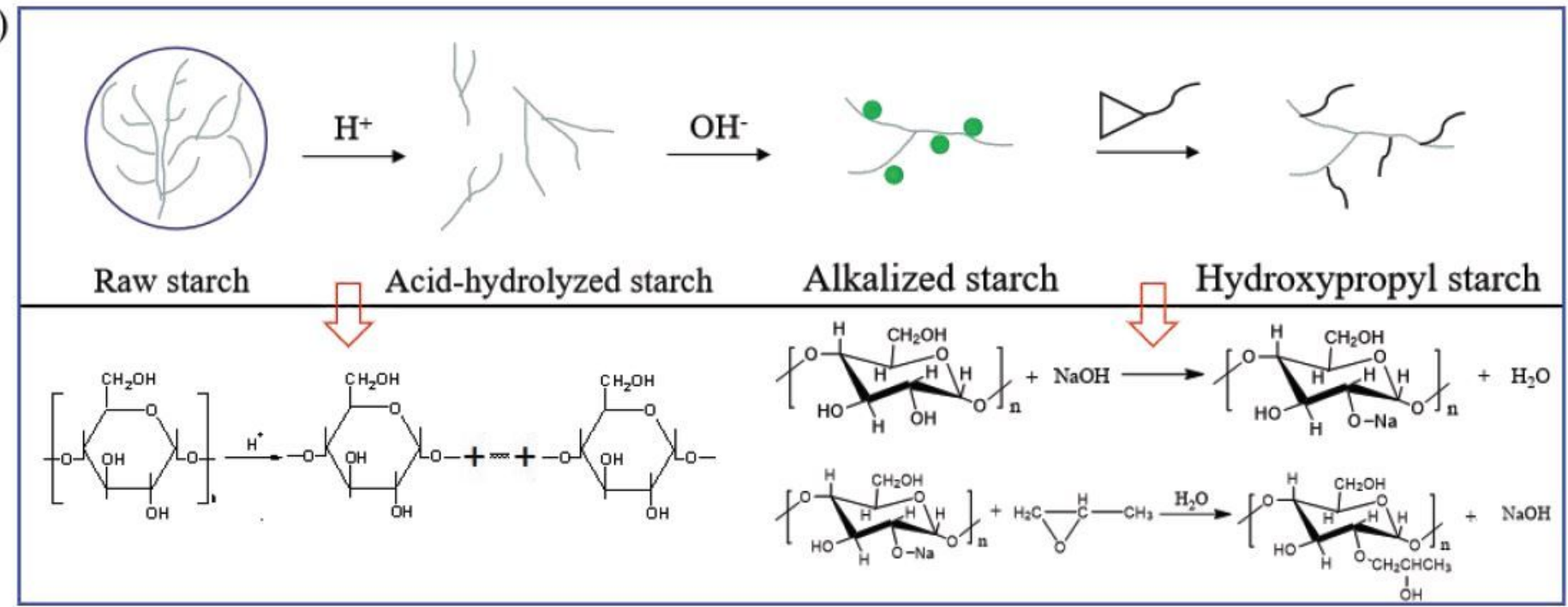

(b)

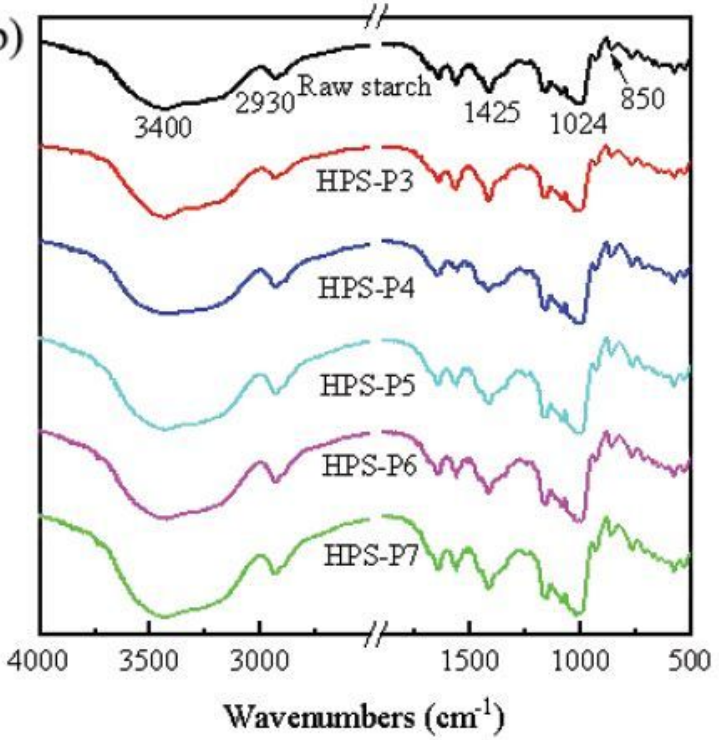

(c)
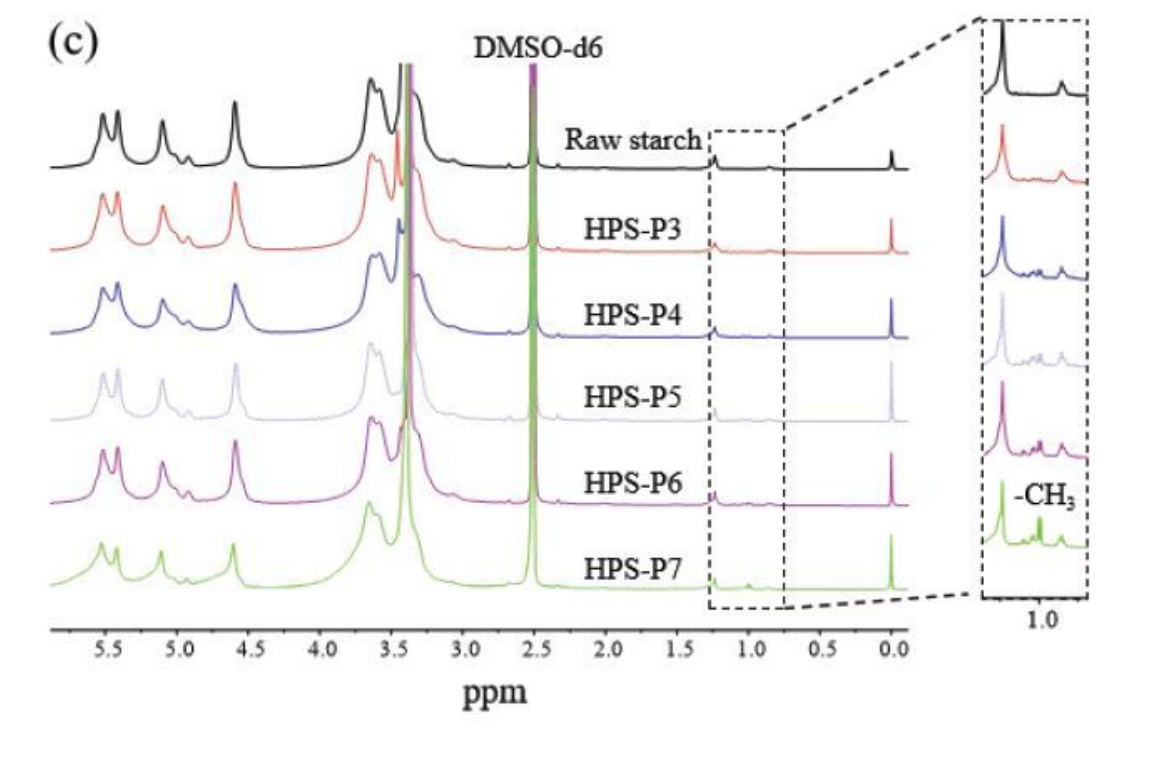

ppm

Figure 1

(a) Preparation of the water-soluble HPS; (b) FTIR and (c) 1H NMR spectra of the raw starch and HPS with different amounts of PO. 

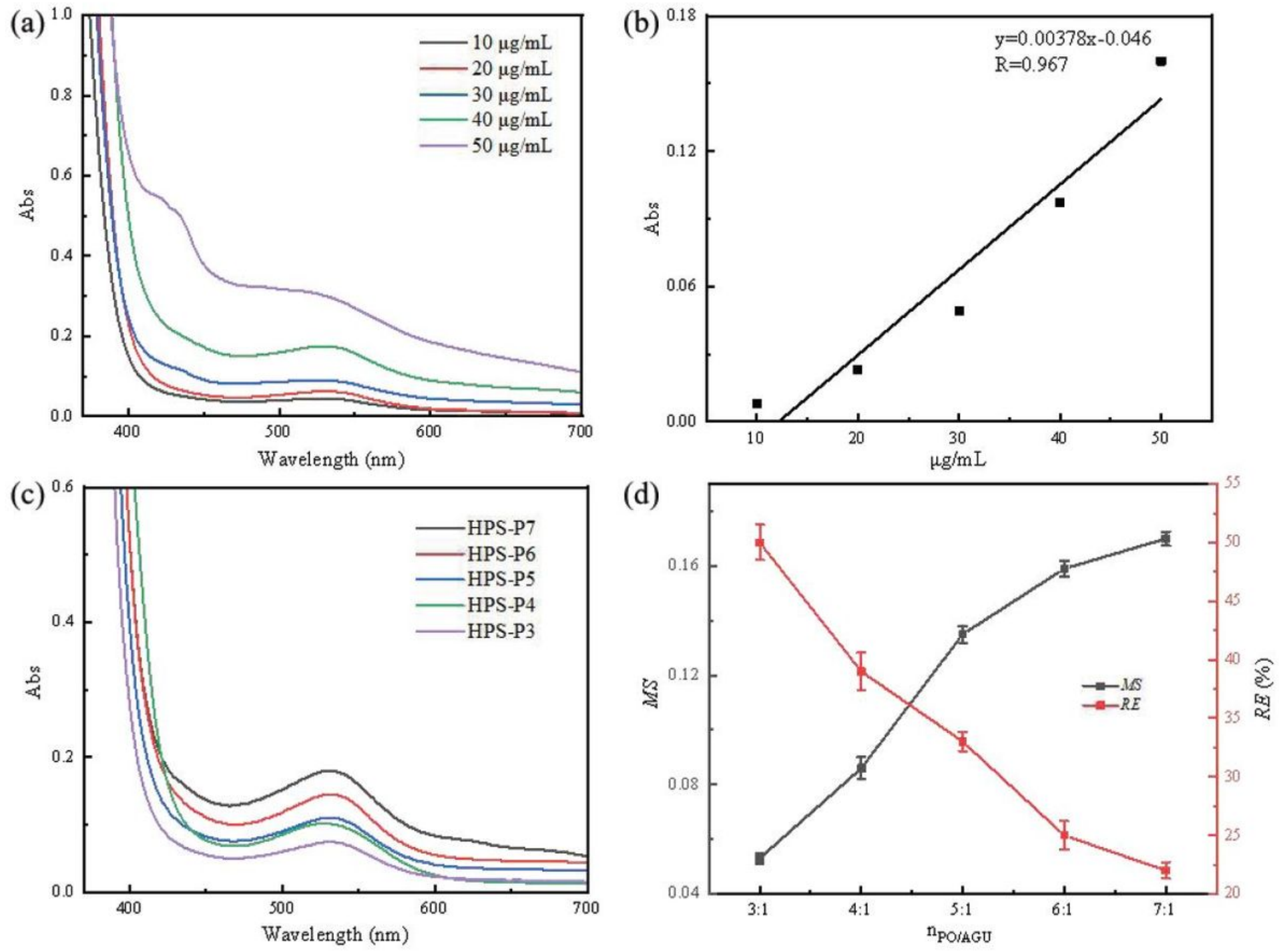

Figure 2

(a) UV-vis spectra of propylene glycol with different concentrations $(10 \sim 50 \mu \mathrm{g} / \mathrm{mL})$; (b) Calibration curve of propylene glycol; (c) UV-vis spectra of HPS samples; (d) The effect of the molar ratio of PO to anhydroglucose unit of starch on MS and the reaction efficiency (RE) of HPS. 


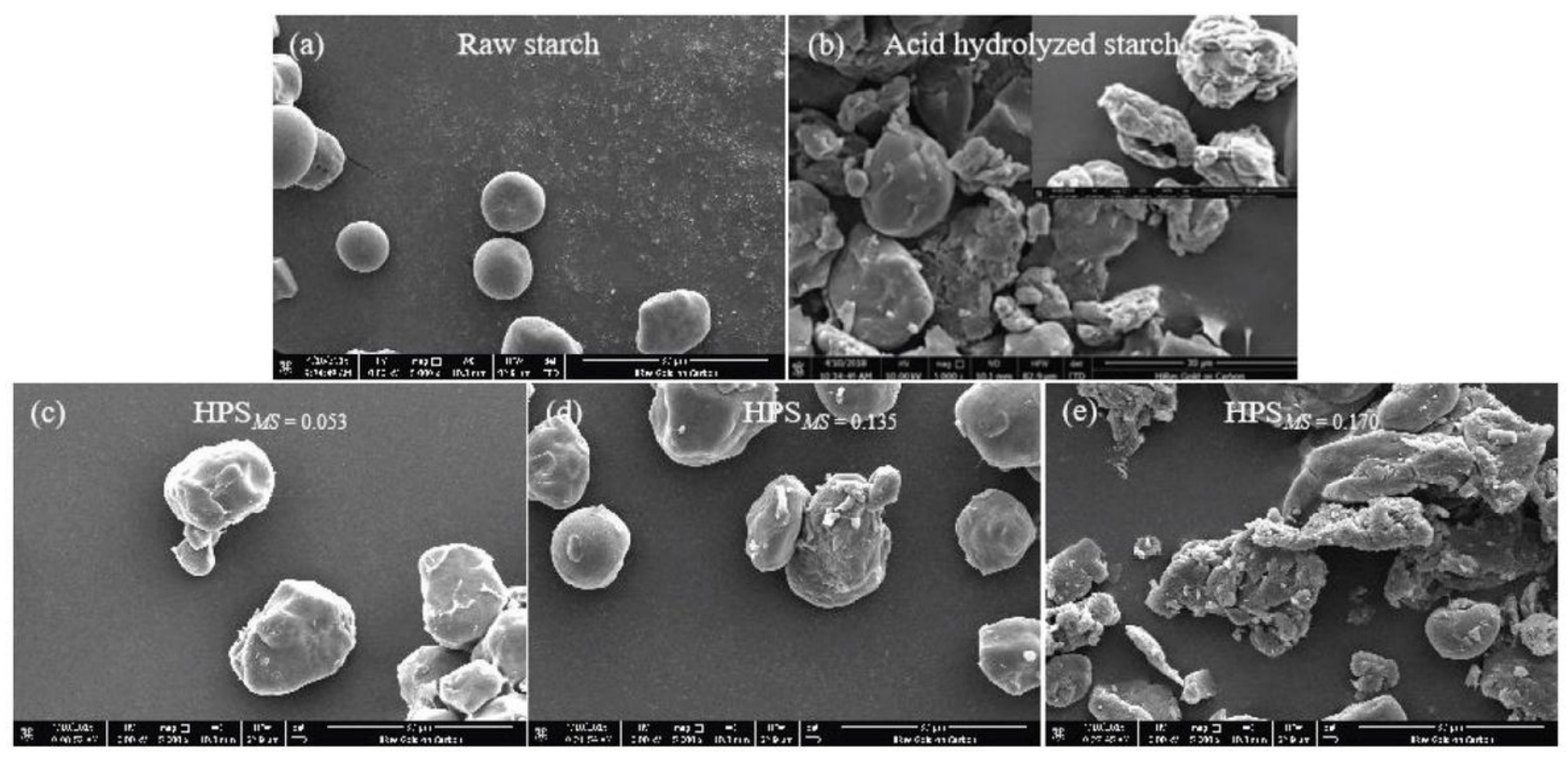

Figure 3

SEM images of the raw starch, acid hydrolyzed starch and HPS with different MS. 


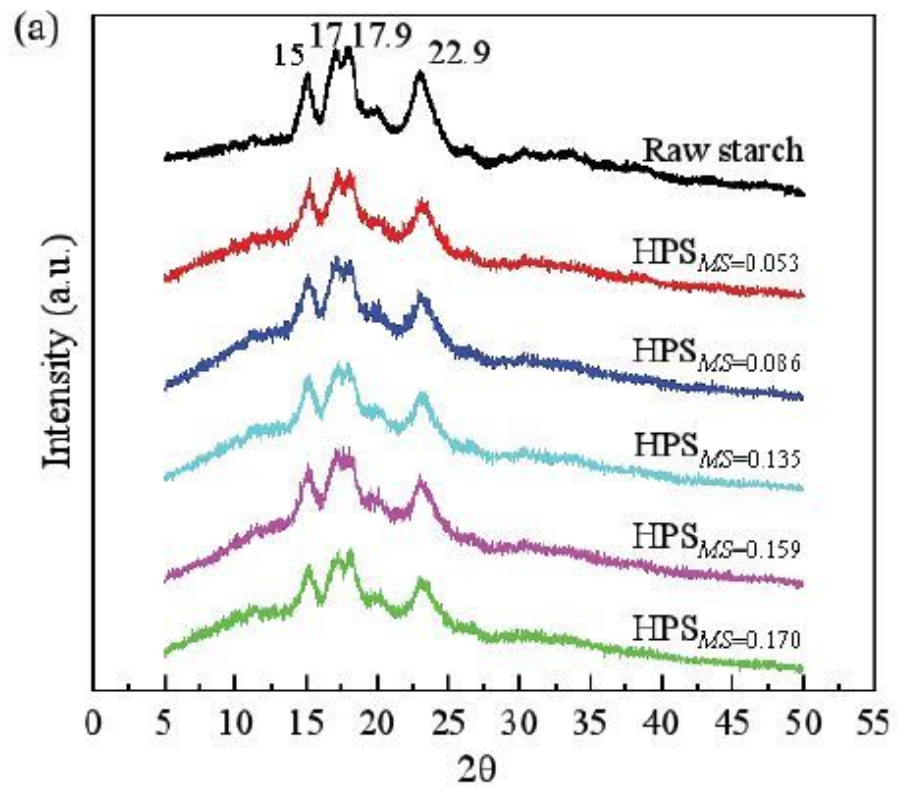

(b)

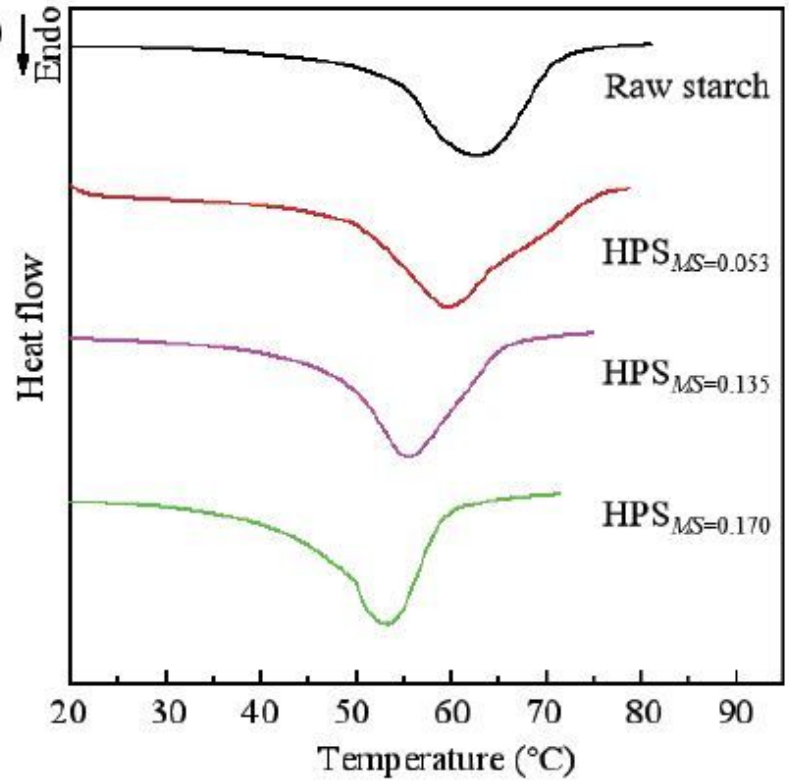

(c)
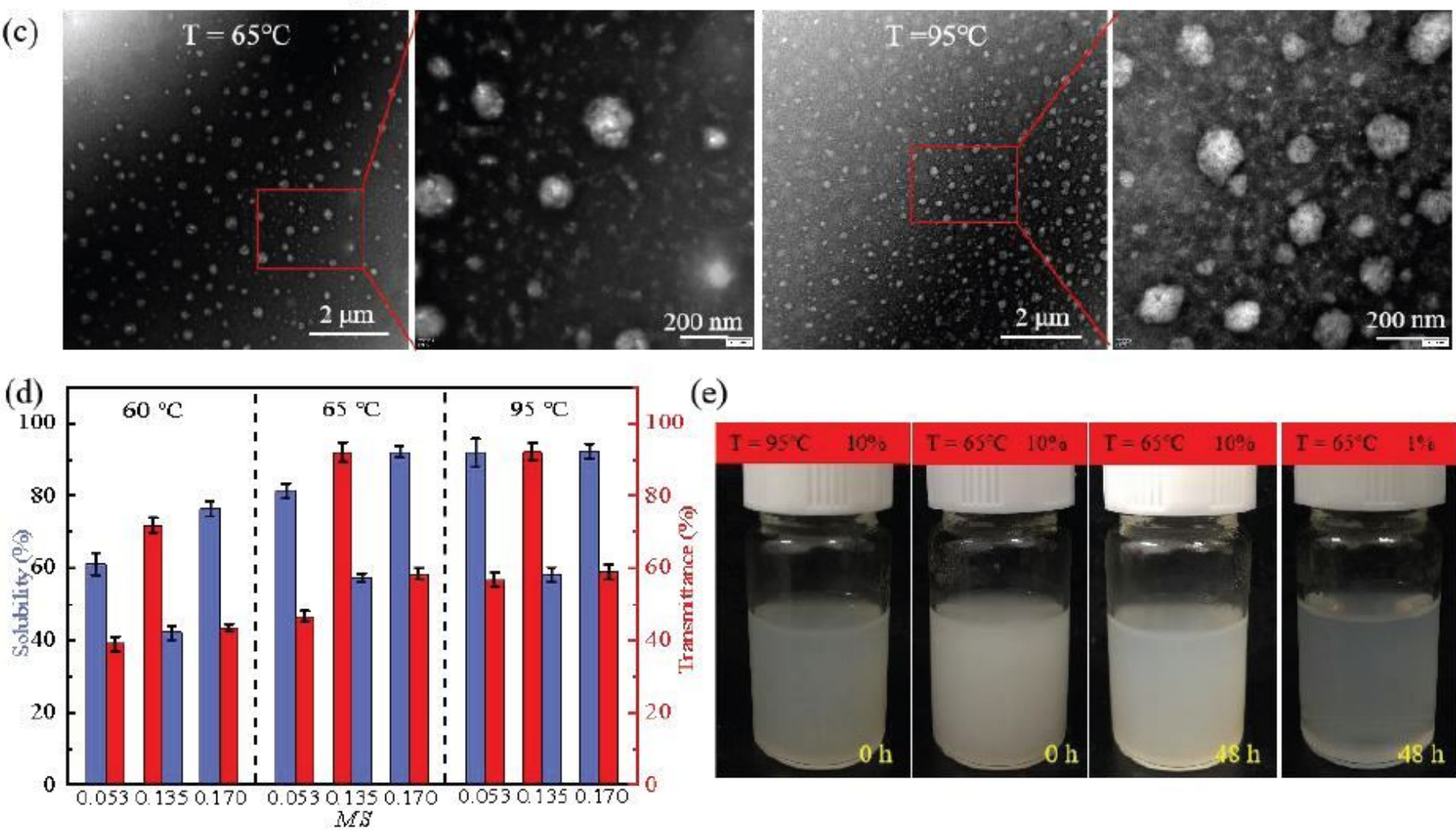

(e)

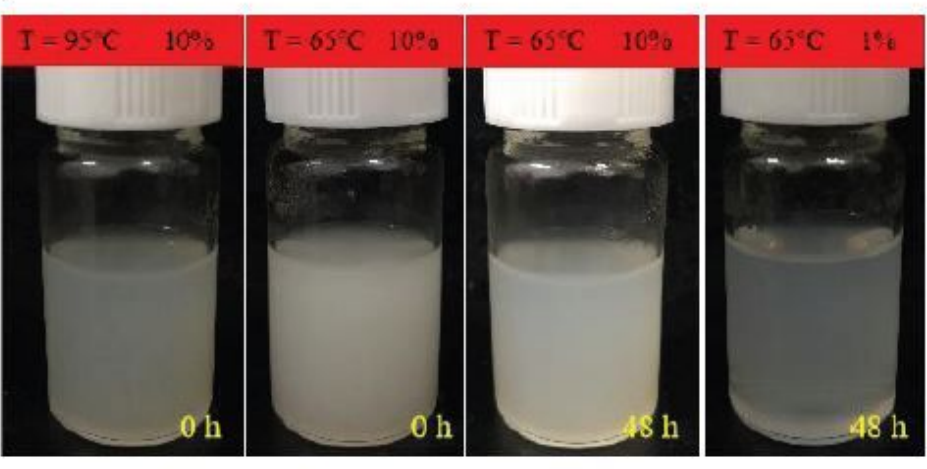

Figure 4

(a) XRD patterns of the raw starch and HPS with different MS; (b) DSC spectra of the raw starch and HPS with different MS; (c) TEM photographs of HPSMS $=0.170$ dissolved at $65^{\circ} \mathrm{C}$ and $95^{\circ} \mathrm{C}$; (d) Comparison of solubility and transmittance of HPS with different MS at $60 \otimes, 65 \otimes$, and $95 \otimes$, respectively; (e) Storage and dilution stability of HPSMS $=0.170$ dissolved at $65 \rrbracket$ and $95 \rrbracket$, respectively. 


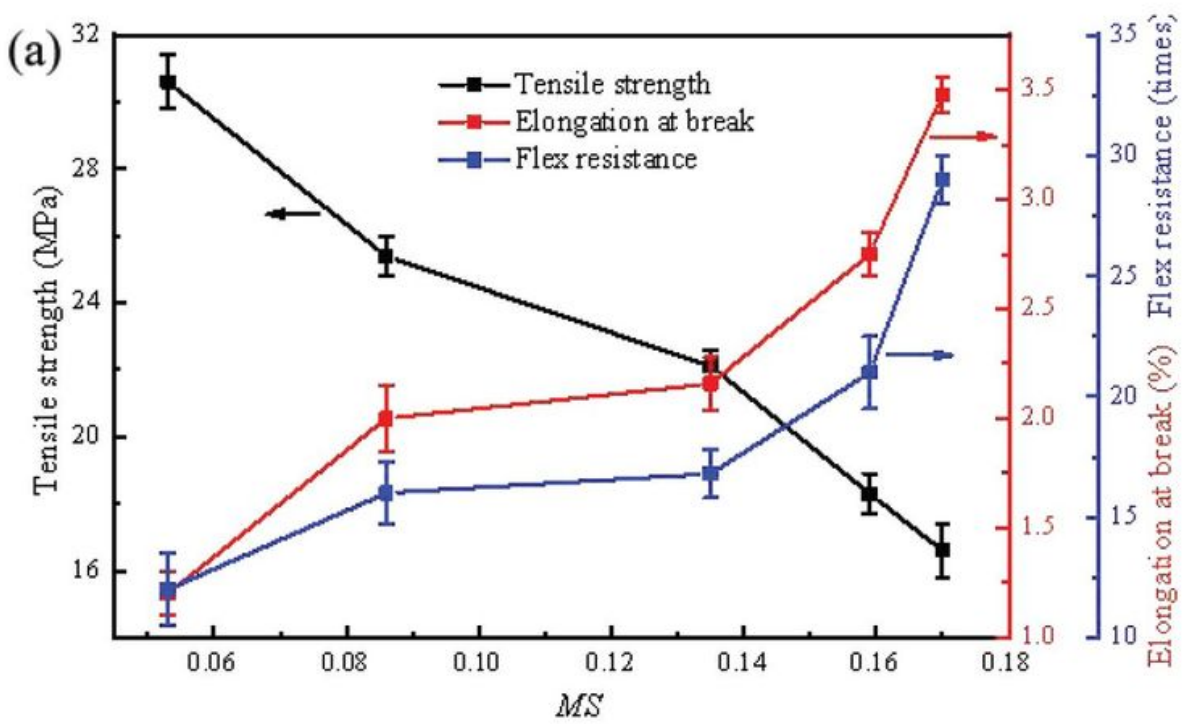

(b)

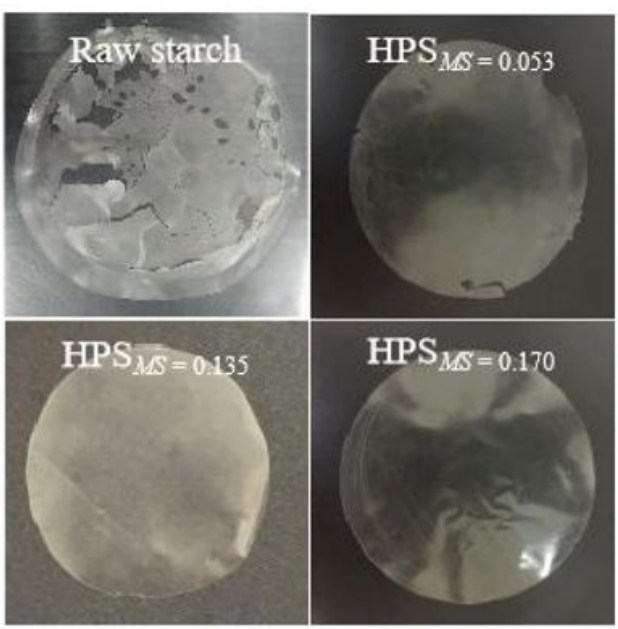

Figure 5

(a) Mechanical properties of HPS films with different MS; (b) Appearance photographs of raw starch and medium temperature water-soluble HPS films.

\section{Supplementary Files}

This is a list of supplementary files associated with this preprint. Click to download.

- graphicsabstract.jpg 